\title{
An Approximation Algorithm for Solving a Class of Minimax Problem
}

\author{
Yingchun Zheng \\ College of Science, Xi'an University of Science and Technology, \\ Xi'an 710054, China \\ Zhychun1979@163.com
}

\begin{abstract}
Based on the the characteristics of maximal function, the algorithm for solving Min-max problem was researched in this paper. A new differential approximation function for the nondifferentiability of the objection function was also constructed. At same time, the property of the new differential approximation function is discussed and the processes of proves show that using the new approximation function to solve the nonlinear unconstrained min-max problem is feasible and effective. The preliminary numerical example shows that the algorithm is effective, and that have a large of convergence characteristics.
\end{abstract}

Keywords: nondifferentiable optimization, Min-max problem, algorithm

\section{Introduction}

An unconstrained nonlinear Min-max problem is usually defined as

$\min _{x \in R^{n}} \varphi(x)$

where

$$
\varphi(x)=\max _{1 \leq i \leq m}\left\{f_{i}(x)\right\}
$$

In general, $f_{i}(x)(i=1,2, \cdots, m)$ is a smooth real-valued function defined on $R^{n}$, where $R^{n}$ is the $\mathrm{n}$-dimensional Euclidean space.$\varphi(x)$ is called the maximum function.

There are a number of different algorithms on the nonlinear Minimax problem. All these algorithms can be classified into three categories [1]:

The first algorithm is implemented by solving the equivalent-linear programming problem [2].But the algorithm is inefficient because of the loss of the original properties of unconstrained optimization.

The second algorithm is suggested to smoothly approximate the maximum function and solve the unconstrained optimization [3-7]. Such this algorithm keeps the unconstrained properties of Min-max problems that its efficiency is high for some appropriate approximate functions.

The third algorithm is presented by constructing the subdifferential function's convex hull [8-9]. Since the subdifferential set is difficult to determine, the algorithms is hardly achieved.

This article will seek a new approximation function and study its characters. The nondifferentiable problems could be converted to the smooth issue, if the maximum function is approach by the new approximation function. Hence, the constrained Min-max problems can be solved by a new algorithm, whose characteristics are simplicity, fast convergence rate, and global convergent. Finally, some numerical examples show that the method is feasible and effective. 


\section{Derivation and Properties of Approximation Function}

The approximation function is constructed as follows:

$$
F_{p}(x)=\frac{\sum_{i \in M} f_{i}^{p+1}(x)}{\sum_{i \in M} f_{i}^{p}(x)}
$$

where $M=\{1,2, \cdots, m\}, f_{i}(x) \geq 0(i \in M)$, and $p \geq 1$.

The following lemmas discuss the properties of the approximation function and are used to prove that the proposing function can precisely approach the maximum function.

Lemma 2.1 If $f_{i}(x)(i \in M)$ is smooth in $R^{n}$, then $F_{p}(x)=\frac{\sum_{i \in M} f_{i}^{p+1}(x)}{\sum_{i \in M} f_{i}^{p}(x)}$ is also smooth in $R^{n}$.

Lemma 2.2 Assume $p \geq 1$, fixed $x$, then $F_{P}(x)$ on $p$ is strictly monotonically increasing.

Proof For some convenience, denoted $f_{i}(x)=f_{i}$, then

$$
\begin{aligned}
& \frac{d F_{P}(x)}{d p}=\frac{\sum_{i \in M} f_{i}{ }^{p+1} \ln f_{i} \sum_{i \in M} f_{i}{ }^{p}-\sum_{i \in M} f_{i}{ }^{p+1} \sum_{i \in M} f_{i}{ }^{p} \ln f_{i}}{\left(\sum_{i \in M} f_{i}{ }^{p}\right)^{2}} \\
& =\frac{\sum_{i, j \in M}\left(f_{i}^{p+1} \ln f_{i}\right) f_{j}{ }^{p}-\sum_{i, j \in M} f_{i}^{p+1} f_{j}{ }^{p} \ln f_{j}}{\sum_{i, j \in M} f_{i}{ }^{p} f_{j}{ }^{p}}
\end{aligned}
$$

In (2-2), because of the symmetry, we can exchange $i$ and $j$, then

$\frac{d F_{P}(x)}{d p}=\frac{\sum_{i, j \in M}\left(f_{i} f_{j}\right)^{p} f_{j}\left(\ln f_{j}-\ln f_{i}\right)}{\sum_{i, j \in M}\left(f_{i} f_{j}\right)^{p}}$

It is note that $\left(f_{i}-f_{j}\right) \times\left(f_{i}-f_{j}\right)>0$. Together this with (2-2) and (2-3), thus

$$
\frac{d F_{P}(x)}{d p}=\frac{1}{2} \frac{\sum_{\substack{i, j \in M \\ i \neq j}}\left(f_{i} f_{j}\right)^{p}\left(f_{i}-f_{j}\right)\left(\ln f_{i}-\ln f_{j}\right)}{\sum_{i, j \in M}\left(f_{i} f_{j}\right)^{p}}>0
$$

\section{Lemma2.3}

$$
m^{-1} \max _{i \in M}\left\{f_{i}(x)\right\} \leq F_{p}(x)=\frac{\sum_{i \in M} f_{i}^{p+1}(x)}{\sum_{i \in M} f_{i}^{p}(x)} \leq \max _{i \in M}\left\{f_{i}(x)\right\} .
$$

Proof By some algebra, we get 
and

$$
\begin{aligned}
& F_{p}(x)=\frac{\sum_{i \in M} f_{i}^{p+1}(x)}{\sum_{i \in M} f_{i}^{p}(x)} \\
& =\frac{\sum_{i \in M} f_{i}^{p}(x) f_{i}(x)}{\sum_{i \in M} f_{i}^{p}(x)} \\
& \leq \frac{\max _{i \in M}\left\{f_{i}(x)\right\} \sum_{i \in M} f_{i}^{p}(x)}{\sum_{i \in M} f_{i}^{p}(x)} \\
& =\max _{i \in M}\left\{f_{i}(x)\right\}
\end{aligned}
$$

$$
\begin{aligned}
& F_{p}(x)=\frac{\sum_{i \in M} f_{i}^{p+1}(x)}{\sum_{i \in M} f_{i}^{p}(x)} \\
& \geq \frac{\sum_{i \in M} f_{i}^{p+1}(x)}{m\left\{\max _{i \in M}\left\{f_{i}(x)\right\}\right\}^{p}} \\
& \geq \frac{\left\{\max _{i \in M}\left\{f_{i}(x)\right\}\right\}^{p+1}}{m\left\{\max _{i \in M}\left\{f_{i}(x)\right\}\right\}^{p}} \\
&=m^{-1} \max _{i \in M}\left\{f_{i}(x)\right\}
\end{aligned}
$$

Lemma 2.4 can be immediately followed by Lemma 2.3 .

Lemma 2.4 If $f_{i}(x)(i \in M)$ is bounded in $R^{n}$, then $F_{p}(x)$ is uniformly bounded in $R^{n}$.

Combination of lemma 2.2, Lemma 2.4 and the principle of monotonic bounded, Theorem 2.1 is obtained.

Theorem 2.1 $F_{p}(x)$ is convergent, namely the limit of $F_{p}(x)$ exists, as $p \rightarrow+\infty$.

By the fact of the uniqueness of the limit of function, we have

Theorem 2.2 $\lim _{p \rightarrow+\infty} F_{p}(x)=\lim _{p \rightarrow+\infty} \frac{\sum_{i \in M} f_{i}^{p+1}(x)}{\sum_{i \in M} f_{i}^{p}(x)}=\max _{i \in M}\left\{f_{i}(x)\right\}$.

The maximum function is approximated by $\left[\sum_{i \in M} f_{i}^{p}(x)\right]^{\frac{1}{p}}$ in literature [10], while is replaced by $\frac{\sum_{i \in M} f_{i}^{p+1}(x)}{\sum_{i \in M} f_{i}^{p}(x)}$ in this paper. Compared to exponential calculation, the 
division operations is much simpler. Hence, the new approximation function supposed by us is more suitable.

\section{Algorithm and its Convergence}

Based on previous discussions, we construct a kind of global convergence of approximating algorithm for solving Min-max problem.

For convenience, we denote as:

$$
\begin{gathered}
M_{f}(x)=\varphi(x)=\max _{1 \leq i \leq m}\left\{f_{i}(x)\right\} \\
f^{*}=\min _{x \in R^{n}} \max _{1 \leq i \leq m}\left\{f_{i}(x)\right\} \\
x^{*}=\left\{x \mid M_{f}(x)=f^{*}\right\} \\
M(x, \xi)=\max _{1 \leq i \leq m}\left\{f_{i}(x)-\xi\right\}=M_{f}(x)-\xi \\
K=\left\{\begin{array}{l}
J(x, \xi)=\left\{i \mid f_{i}(x)-\xi \geq 0, i \in M\right\}, M(x, \xi)>0 \\
M, M(x, \xi) \leq 0
\end{array}\right. \\
U(x, \xi)=\frac{\sum_{i \in K}\left(f_{i}(x)-\xi\right)^{p+1}}{\sum_{i \in K}\left(f_{i}(x)-\xi\right)^{p}}
\end{gathered}
$$

It is clear that only when the $\xi=0$, then $U(x, \xi)=F_{p}(x)$, thus $U(x, \xi)$ is a reasonable

deformation of $F_{P}(x)$. Now, we present the algorithm 1 .

\section{Algorithm 1}

Step1: Given the initial point $x^{0} \in R^{n}$, the accuracy of $\varepsilon, \xi_{1}=\min \left[0, M_{f}\left(x_{0}\right)\right]$, $k=1$, select the parameter $p>1$.

Step2: Solve the unconstrained optimization problems $\min _{x \in R^{n}} U\left(x, \xi_{k}\right)$, whose solution is $x^{k}$.

Step3: Let $\xi_{k+1}=M_{f}\left(x^{k}\right)$.

Step4: (Stop condition) If $\left|\xi_{k+1}-\xi_{k}\right|$, stop; otherwise, let $k=k+1$, and return to step2.

The feature of algorithm 1 is that the maximum value is forced to departure the level $\xi_{k}$ under the k'st iteration. When $\mathrm{k}<2$, we get $M\left(x^{k}, \xi_{k}\right)<0$ and $\xi_{k+1}<\xi_{k}$. Following theorem will deal with the convergence of the algorithm 1 . 
Lemma 3.1 If $k>2$, thus $\left|U\left(x^{k}, \xi_{k}\right)\right| \geq\left|U\left(x^{k+1}, \xi_{k+1}\right)\right|$.

Theorem 3.1 If $k \rightarrow \infty$, thus $\left|U\left(x^{k}, \xi_{k}\right)\right| \rightarrow 0$.

Proof If $k>2$, then

and

$$
\begin{aligned}
|U(x, \xi)| & =\left|\frac{\sum_{i \in M}\left(f_{i}\left(x^{k}\right)-\xi_{k}\right)^{p+1}}{\sum_{i \in M}\left(f_{i}\left(x^{k}\right)-\xi_{k}\right)^{p}}\right| \\
= & \frac{\left|\sum_{i \in M}\left(f_{i}\left(x^{k}\right)-\xi_{k}\right)^{p+1}\right|}{\left|\sum_{i \in M}\left(f_{i}\left(x^{k}\right)-\xi_{k}\right)^{p}\right|} \\
& \leq\left|\max _{i \in M}\left(f_{i}\left(x^{k}\right)-\xi_{k}\right)\right| \\
& =\left|\max _{i \in M}\left\{f_{i}\left(x^{k}\right)\right\}-\xi_{k}\right| \\
& =\left|\xi_{k+1}-\xi_{k}\right| \\
& =\xi_{k}-\xi_{k+1}
\end{aligned}
$$

$$
\lim _{i \rightarrow \infty} \sum_{k=2}^{i}\left|U\left(x^{k}, \xi_{k}\right)\right| \leq \lim _{i \rightarrow \infty}\left(\xi_{2}-\xi_{i+1}\right) \leq \xi_{2}=M_{f}\left(x^{1}\right)
$$

Then,

$$
\lim _{i \rightarrow \infty}\left|U\left(x^{k}, \xi_{k}\right)\right|=0
$$

Theorem 3.2 If $k \rightarrow \infty$, thus $M_{f}\left(x^{k}\right) \rightarrow M_{f}\left(x^{\infty}\right)=f^{*}$.

Proof Assuming $M_{f}\left(x^{k}\right) \rightarrow L$, we just to probe $L=M_{f}\left(x^{\infty}\right)$. If it do not hold, there is $L>M_{f}\left(x^{\infty}\right)$.

Because $M_{f}\left(x^{k}\right)$ is a continuous function with respect to $x$, there is point $\bar{x}$, thus

$$
M_{f}\left(x^{\infty}\right)<M_{f}(\bar{x})<L, f_{i}(\bar{x})-L<0, i \in M
$$

If $k \rightarrow \infty, U\left(x^{k}, \xi_{k}\right) \rightarrow 0$,

thus

$\lim _{k \rightarrow \infty} \xi_{k}=L$.

while

$$
\begin{gathered}
U(\bar{x}, L)=\frac{\sum_{i \in K}\left(f_{i}(x)-L\right)^{p+1}}{\sum_{i \in K}\left(f_{i}(x)-L\right)^{p}} \\
=\frac{(-1)^{p+1} \sum_{i \in K}\left(L-f_{i}(x)\right)^{p+1}}{(-1)^{p} \sum_{i \in K}\left(L-f_{i}(x)\right)^{p}}<0,
\end{gathered}
$$


This is contradiction that $x^{k}$ is the minimum point of $U(x, L)$ if $k \rightarrow+\infty$.

Together with Theorem 3.1 and Theorem 3.2, we obtain $\left|U\left(x^{k}, \xi_{k}\right)\right| \rightarrow 0$ and $M_{f}\left(x^{k}\right) \rightarrow f^{*}$ if $k \rightarrow+\infty$.

The main features of algorithm 1 are: First, there is no constriction on the initial point, and it is a large-scale convergence of the algorithm; Second, the algorithm belongs approximation algorithm. Therefore, the singularity problem and non-singular problems can be treated equally; Third, the parameters $p$ can be chosen finite value, by which the optimal solution with high accuracy can be achieved.

Numerical experiments explicitly show that the algorithm can quickly convergent at the beginning of iteration. However, when iterative point $x^{k}$ approximates the point of optimal value $x^{*}$, the rate of convergence is slower. The reason is that, on one hand, the algorithm is influenced by the rounding errors; on the other hand, we do not necessarily need to approach such a complex form near the optimum points $x^{*}$, and can consider other simpler approximation. Hence, the two-stage accelerateing algorithms are given as follows.

Algorithm 2 (given initial approximation $x_{0}$ )

[First stage](Adopt estimate in algorithm 1)

Step 1: Let $B_{l}=\frac{M_{f}\left(x^{r}\right)+\xi_{r}}{2}$;

Step 2: Let $\varphi_{1}=B_{l}, k=r$;

Step 3: Solve unconstraint sub-problems $\min _{x \in R^{n}} p\left(x, \varphi_{k}\right)$ with its solution $x^{k}$;

[Second stage]( Adopt Dutta Accelerating algorithms)

Step 4: Let $B_{u}=\min \left(M_{f}\left(x^{r}\right), 10\right)$;

Step 5: Calculation

$$
\begin{aligned}
& M^{m}=\varphi_{k}+\left\{\frac{p\left(x^{k}, \varphi_{k}\right)}{m}\right\}^{1 / 2}, \\
& M^{i}=\varphi_{k}+\frac{p\left(x^{k}, \varphi_{k}\right)}{\sum\left[f_{i}\left(x^{k}\right)-\varphi_{k}\right]}, i \in I\left(x^{k}\right)
\end{aligned}
$$

where $I\left(x^{k}\right)=\left\{i \mid f_{i}\left(x^{k}\right)>\varphi_{k}\right\}$;

Step 6: If $M^{i}<B_{u}$, let $M^{i}<B_{u}$; if not, let $\varphi_{k+1}=M^{m}$;

Step 7: Let $\varphi_{0}=\varphi_{k+1}-\varphi_{k}, B_{l}=M^{m}, S=p\left(x^{k}, \varphi_{k}\right), k=k+1$, its solution for $x^{k}$;

Step 8: Solve unconstraint sub-problems $\min _{x \in R^{n}} p\left(x, \varphi_{k}\right)$ with its solution $x^{k}$;

Step 9: If $\left(B_{u}-B_{l}\right)<\varepsilon$ or $\varphi_{0}<\varepsilon$, then stop;

Step 10: $\quad$ If $p\left(x^{k}, \varphi_{k}\right)>S$, return to Step5; otherwise, if $S<S m a l$, stop; if not,

let $B_{u}=\varphi_{k}, S=0, \varphi_{k}=B_{l}$, return to Step8. 
In algorithm 2 [second stage], we let $p(x, \varphi)=\sum_{i \in I(x)}\left(f_{i}(x)-\varphi\right)^{2}$, where $p(x, \varphi)$ is simpler than $F_{P}(x)$ to approach the constructed $U(x, \xi)$. The parameter $\varepsilon, \xi$ and Smal are provided for stopping.

Combination of algorithm 1 and Dutta, algorithm 2 not only has the advantage of globally convergent, but is fast rate of the overall convergence.

\section{Numerical Experiments}

A numerical experiment is executed. In order to solve unconstrained sub-problem in the above Algorithm 1 and Algorithm 2, we use variable metric method (DFP or BFGS method) and the conjugate gradient method (PRP method) to conduct the numerical experiments.

The numerical results show that the BFGS algorithm is appropriate. Therefore, this article adopts s a standard BFGS algorithm subroutine.

Experimental function 1:

$$
\begin{gathered}
f_{1}(x)=x_{1}{ }^{2}+x_{2}{ }^{4}, \\
f_{2}(x)=\left(2-x_{1}\right)^{2}+\left(2-x_{2}\right)^{2}, \\
f_{3}(x)=2 \exp \left(-x_{1}+x_{2}\right)
\end{gathered}
$$

Experimental function 2:

$$
\begin{gathered}
f_{1}(x)=x_{1}{ }^{2}+x_{2}{ }^{4}+x_{1} x_{2}, \\
f_{2}(x)=\sin x_{1}, \\
f_{3}(x)=\cos x_{2}
\end{gathered}
$$

Experimental function 3:

$$
\begin{gathered}
f_{1}(x)=x_{1}{ }^{4}+x_{2}{ }^{2}, \\
f_{2}(x)=\left(2-x_{1}\right)^{2}+\left(2-x_{2}\right)^{2}, \\
f_{3}(x)=2 \exp \left(-x_{1}+x_{2}\right)
\end{gathered}
$$

Through a variety of numerical experiments, the algorithm 2 is ideal algorithm to solve the Min-max problem.

First of all, the numerical results for different initial point of each experiment function are collected in Table 1

Table 1. Experimental Results of Different Initial Points

\begin{tabular}{|c|c|c|c|c|}
\hline $\begin{array}{l}\text { Experimenta } \\
\text { function }\end{array}$ & initial points & Parameter $\mathrm{p}$ & Iterations & Accuracy \\
\hline 2 & $(2,1)$ & 1000 & 9 & $10^{-6}$ \\
\hline 2 & $(10,6)$ & 120 & 9 & $10^{-6}$ \\
\hline 2 & $(100,70)$ & 200 & 10 & $10^{-6}$ \\
\hline 2 & $(1000,1000)$ & 300 & 10 & $10^{-6}$ \\
\hline 3 & $(1,-0.1)$ & 1000 & 10 & $10^{-6}$ \\
\hline 3 & $(2,2)$ & 100 & 8 & $10^{-6}$ \\
\hline
\end{tabular}

For experimental function 3, the compare between the new algorithms and existing algorithms is excised. The numerical results are shown in table 2 . 
Table 2. Comparison of the Numerical Results of Different Algorithms

\begin{tabular}{|c|c|c|c|c|}
\hline $\begin{array}{l}\text { Experimenta } \\
\text { function }\end{array}$ & $\begin{array}{c}\text { initial } \\
\text { points }\end{array}$ & algorithm & Iterations & Accuracy \\
\hline 3 & $(3,1)$ & D.H.Anderson[3] & 10 & $10^{-3}$ \\
\hline 3 & $(3,1)$ & K.Madsen[5] & 20 & $10^{-3}$ \\
\hline 3 & $(3,1)$ & Algorithm 1 & 9 & $10^{-6}$ \\
\hline 3 & $(3,1)$ & Algorithm 2 & 5 & $10^{-6}$ \\
\hline
\end{tabular}

Discussion on advantages and differences:

Table 1 illustrates the algorithm is not sensitive for the initial point, and shows that this algorithm has the properties of global convergence.

Table 2 presents the features of this algorithm is fast convergence and less iteration.

These results show this new algorithm is not only fast convergence, but maintains a wide range of convergence characteristics. It is interesting that the function is not negative, but the function could be negative to constructed approximation function to solve problem in actual problem. If $g_{i}(x)=f_{i}(x)+G$, where $G$ is a large positive number, then $g_{i}(x)$ is non-negative. Thus $\min _{x \in R^{n}} \max _{1 \leq i \leq m}\left\{g_{i}(x)\right\}$ and $\min _{x \in R^{n}} \max _{1 \leq i \leq m}\left\{f_{i}(x)\right\}$ have the same optimal solution.

\section{Conclusion}

For Min-max problems, maximum function is generally not differentiable. In this paper, we construct a new smooth function to approximate maximum function. The approximation function is simple and easy to calculate. Thus, a kind of convergence for solving Min-max problems with a wide range of algorithms is suggested and numerical results show that the methods are effective. Along with the gradually increase of the parameter $p$, approximation function $F_{P}(x)$ gradually approach the maximum function. Since incensement of parameter $p$ makes the function value overflow, it is very important to select the appropriate parameter. In addition, when the iterative $x_{k}$ tends to the optimal value $x^{*}$, the rate of convergence is slow. Therefore, our algorithm should be adopted at the beginning of these steps. When the iteration point $x_{k}$ approaches to the optimal value of the point $x^{*}$, the effect of using cutting plane method or sub-gradient method is better.

\section{Acknowledgment}

This work is supported by National Natural Science Foundation of China under Grant No. 71103143,No. 71473194; Science and Technology Foundation of Shaanxi Province of China under Grant No. 2013XJXX-40.

\section{References}

[1] N. Z. Shor, Editor, Minimization Methods for Nondiffenrentiable Function, New York Springer-Verlag, New York, (1979).

[2] E. Polak and J. O. Royset, "On the Use of Augmented Lagrangians in the Solution of Generalized SemiInfinite Min-Max Problems", Computational Optimization and Applications, vol.31, no.2,(2005).

[3] F.S. Wang and J.B. Jian, "A nonmonotonic hybrid algorithm for min-max problem", Optimization and Engineering,vol.15,no.4,(2014).

[4] R. Laraki and J. B. Lasserre, "Semidefinite programming for min-max problems and games" Mathematical Programming,vol.131,no.1,(2012). 
[5] G. Gutoski and X. D. Wu, "Parallel Approximation of Min-Max Problems", Computational Complexity, vol.22,no.2, (2013).

[6] A. Dolgui and S. Kovalev, "Min-max and min-max (relative) regret approaches to representatives selection problem", 4OR, vol.10,no.2 (2012).

[7] C. Y. Ren, "Fast Taboo Search Algorithm for Solving Min-Max Vehicle Routing Problem", Communications in Computer and Information Science, vol.236,no.2, (2011).

[8] X. F. Wang, "an algorithm forsolving nondifferentiable programming on circumscribed rectangular parallelepiped of subdifferentiable", Journal of Northwest University,vol.39,no.2,(2009).

[9] S. Gaubert and W. M. McEneaney, "Min-Max Spaces and Complexity Reduction in Min- Max Expansions", Applied Mathematics \& Optimization, vol.65, no.3,(2012).

[10] H. X. Wen, "A New Algorithm for solving unconstrained Min-max problem", Journal of Xidia University,vol.16,no.2,(1989).

\section{Author}

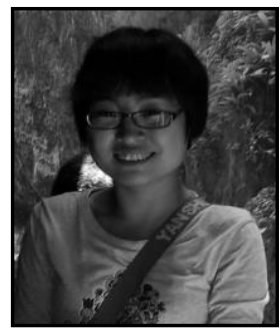

Yingchun Zheng. Author's profile: Yingchun Zheng was born in Shaanxi Province, China on April 9, 1979. She received his master's degree in Mathematics from Northwest University University, China in 2002. Now She is an associate professor of School of Science, Xi'an University of Science and Technology, Xi'an, China. Her main research interests include Optimization Method and Algorithm. 
International Journal of Grid and Distributed Computing

Vol. 9, No. 5 (2016) 